\title{
The influx rate of long-period comets in the Earth's neighborhood and their debris contribution to the interplanetary medium
}

\section{Julio Angel Fernández}

Departamento de Astronomia, Facultad de Ciencias, Igua 4225, 11400 Montevideo, Uruguay email: julio@fisica.edu.uy

\begin{abstract}
We analyze the flux of new and evolved long-period comets (LPCs) reaching the Earth's neighborhood (perihelion distances q $<1.3 \mathrm{AU}$ ), their physical lifetimes, and their implications as regards to the amount of meteoritic matter that is being deposited in the nearEarth region. The flux of LPCs with $\mathrm{q}<1.3 \mathrm{au}$ is found to be of about $340 \pm 40$, brighter than absolute total magnitude 8.6 (radius $\mathrm{R} \sim 0.6 \mathrm{~km}$ ) (Fernández and Sosa 2012). Bearing in mind that most of these comets disintegrate into meteoritic matter, this represents a large contribution to the interplanetary dust complex which requires an amount of matter of about 10 tons $s^{-1}$ to keep it in steady state. These aspects, as well as the impact rate with Earth of meteoroids of LPC origin, will be discussed in this presentation.
\end{abstract}

